\title{
Sounding of Layered Marine Bottom - Model Investigations
}

\author{
G. GrelowsKA ${ }^{a, *}$ AND E. KOZACZKA ${ }^{a, b, c}$ \\ ${ }^{a}$ Polish Naval Academy, Smidowicza 69, 81-103 Gdynia, Poland \\ ${ }^{b}$ Gdansk University of Technology, Narutowicza 11/12, 80-223 Gdańsk, Poland \\ ${ }^{c}$ University of Technology and Life Sciences, Ks. Kordeckiego 20, 85-225 Bydgoszcz, Poland
}

\begin{abstract}
The necessary actions connected with interpretations of examining of physical properties of sea bottom structure is developing of proper procedure of modeling of the layered structure. The implementation of the acoustic waves for the determination of seabed stratification requires a precise diagnosis of the topic from both the theoretical and practical side. Little differences between the parameters of sediments, as well as irregular distance between layers make the interpretation of the images of cross sections uneasy. The paper presents the model of stratified structure of seabed in the form of a liquid on which falls the perpendicular elastic wave. Since the description of the seabed is made on the basis of reflected impulse shape, which provides the information about the structure and material properties such as density and speed of wave propagation, the important role among other things has the spatial resolution, because the pulse length determines the possibility of distinguishing between the layers. These aspects require very careful analysis of the form of reflected signals.
\end{abstract}

PACS numbers: 43.30.Ma, 43.50.Pc, 43.25.Jh

\section{Introduction}

Seabed characterization of shallow water environments is a matter of great interest due to the huge spectrum of potential applications such as hydrography and cartography of littoral regions, military oceanography, marine geosciences, offshore industry, environmental protection, etc.

Interpretation of data obtained during sea bottom structure measurements (Fig. 1), as well as modeling the phenomena of sound propagation in sediments, are challenging tasks due to many factors influencing the phenomena. For solving these problems, three topics within underwater acoustics are of primary interest: the acoustic environment, theories for wave propagation in sediments, and models derived from these theories.

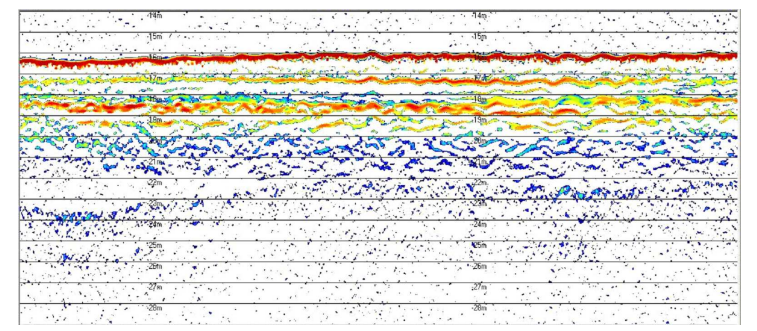

Fig. 1. Layered sub-bottom structure obtained using the parametric echosounder SES [1].

Understanding of the environment is essential for solution of many of the problems in shallow-water acoustics. It requires that properties such as sediment mass density,

* corresponding author; e-mail: g.grelowska@amw.gdynia.pl sound speed and acoustic attenuation is known. Moreover, it requires knowledge of the seafloor volume heterogeneity and surface roughness. Given this information, virtually any acoustic problem can be solved, in principle, because the equations of motion and boundary conditions for the acoustic field are understood. Evidently, computational difficulties may arise; more significantly, there is some uncertainty as to the applicability or accuracy of current theories for wave propagation in sediments. It is also important that sediment properties reflect seafloor conditions at the time of acoustic measurements or modeling applications. Seafloor properties in shallow water are not only spatially variable (heterogeneity) but can also vary with time. For example, seafloor morphology (roughness or ripple structure) changes rapidly in response to wave action during storms and can be subsequently degraded due to biological processes.

The list of geophysical properties, or parameters, of interest is quite long, due to the continual refinement of sediment acoustic theory and models. Acoustic models can be broadly classified according to the wave theory employed e.g. fluid, elastic, or poroelastic. Such theories sometimes require knowledge of sediment structure at the microscopic level, and often require knowledge of the centimeter-scale spatial dependence of the material parameters required by the underlying theory. Such spatial dependence includes the random spatial variation responsible for scattering and the average variation with depth below the seafloor due to stratification.

\section{Sea bottom properties}

Geophysical properties of the sea bottom could be divided into two categories [2]: physical properties and geoacoustic properties. Physical properties include those 
characteristics of sediment as sediment grains and pore fluid are of interest to geologists and geotechnical engineers, as well as having applications to acoustics. These properties include sediment mass density, porosity, permeability, and many other parameters associated with sediment grains, pore fluid, the sediment frame, and the pore space. Geoacoustic properties include speed and attenuation for both compressional and shear waves. Acoustic impedance, the product of sediment mass density and sound speed, is another important geoacoustic property. The properties most commonly used in current acoustic models will be introduced and methods developed to characterize the random roughness and heterogeneity of the seafloor will be described.

The division of geophysical properties into physical and geoacoustic components is motivated in part by efforts to predict geoacoustic properties given measured values of one or more physical properties. Typically, this involves the development of empirical regressions giving wave speed or attenuation in terms of properties such as grain size or porosity.

Some sediment physical properties, such as mass density (referred also as "bulk density"), are used directly as input parameters in acoustic theory and models. Others, such as sediment type or mean grain size, are used indirectly as empirical predictors of acoustic behavior [3]. In many applications of this type of model, a value of mean grain size is all that is required. Grain size is one of the most commonly measured sediment properties, and is usually given as equivalent particle diameter, either in millimeters or in logarithmic units. Logarithmic units are convenient, because many grain-size distributions are approximately lognormal. If the grain size is $d \mathrm{~mm}$, the grain size is recalculated into base-two logarithmic units as follows:

$$
\Phi=-\log _{2} d
$$

In Table, comparison of grain size as well as geoacoustical parameters of main types of sediments is given.

TABLE

Some geophysical parameters of sea bottom sediments [3].

\begin{tabular}{c|c|c|c|c|c|c}
\hline \hline Sediment & Symbol & Grain size $[\mathrm{mm}]$ & $\Phi$ & $\begin{array}{c}\text { Dimensionless } \\
\text { sound speed }\end{array}$ & $\begin{array}{c}\text { Dimensionless } \\
\text { density }\end{array}$ & $\begin{array}{c}\text { Absorption coefficient } \\
{\left[\mathrm{dB} \mathrm{m}^{-1} \mathrm{kHz}^{-1}\right]}\end{array}$ \\
\hline fine gravel & $\mathrm{FGr}$ & 2.0 & -1 & 1.338 & 2.4923 & 0.4556 \\
coarse sand & $\mathrm{CSa}$ & 0.630 & 0.66 & 1.243 & 2.2047 & 0.4718 \\
fine sand & $\mathrm{FSa}$ & 0.20 & 2.32 & 1.0364 & 1.2236 & 0.6958 \\
medium silt & $\mathrm{MSi}$ & 0.0063 & 7.31 & 0.9841 & 1.147 & 0.0763 \\
clay & $\mathrm{Cl}$ & 0.002 & 8.97 & 0.9801 & 1.1449 & 0.0537
\end{tabular}

Particles larger than sand-sized are often referred to as "gravel", while clay- and silt-sized particles are collectively referred to as "mud".

Geoacoustic parameters, such as speed and attenuation of waves propagating in sediment, are the essential inputs to sediment wave propagation theories. It follows that they are also needed for theory-based acoustic models. Acoustic impedance is another frequently encountered geoacoustic parameter which utility stems from its ease of measurement and high degree of correlation with other parameters. While the model builder has the possibility of choosing a certain set of geoacoustic parameters as inputs, the model user faces the problem of obtaining values for these parameters. In a few cases (e.g., Biot theory), some of the geoacoustic parameters can be calculated in terms of physical parameters such as the densities and elastic moduli of the constituents (particles and pore water) as well as sediment bulk properties such as porosity and permeability. Generally, however, one has neither a sufficient set of measured physical parameters nor a sufficiently complete and accurate theory that this approach can be used. Failing this, direct measurement of bulk density, and in situ measurement of wave properties is the preferred method of obtaining geoacoustic parameters. Such detailed geoacoustic measurements are rare, as they require instrumentation and expertise usually available only in carefully planned field experiments. It is more likely that a few physical properties, such as mean grain size and porosity, might be measured. In these cases, one can use empirical regressions to estimate values of geoacoustic parameters.

Fluid theory requires that the sediment be described in terms of three parameters, usually taken to be the bulk density, compressional wave speed, and compressional wave attenuation. Additional geoacoustic parameters are needed if the seafloor is modeled as an isotropic elastic solid supporting shear waves. In this case sediment bulk density and a set of four elastic properties must be known. One such set consists of the compressional and shear wave speeds and corresponding attenuations. Alternately, elastic moduli can be used. Hamilton [4] presents a version of elasticity in which the elastic con- 
stants (shear modulus and bulk modulus) are replaced with complex constants with the real parts governing wave speeds and the imaginary parts governing wave attenuations. Hamilton assumes that the complex moduli are independent of frequency. As a result, attenuation increases linearly with frequency and wave speeds are independent of frequency (negligible speed dispersion). The proportionality constant, $k$, connecting attenuation and frequency is widely encountered in the literature and is usually given in $\mathrm{dB} \mathrm{m}^{-1} \mathrm{kHz}^{-1}$.

Sediments, particularly sands, can be treated as poroelastic media that allow independent movement between the pore fluid and the "frame", comprised of sediment particles in contact. Modeling scattering from a poroelastic seafloor requires several physical parameters, many of which can be inferred from geoacoustic parameters. This is a role reversal compared to fluid modeling, where geoacoustic parameters are the model inputs and are often inferred from physical parameters. The physical parameters required by poroelastic theory can be broken into four sets characterizing the (1) fluid, (2) pore, (3) grain, and (4) frame properties. The fluid parameters are density, viscosity, and bulk modulus. The porometric parameters are porosity, tortuosity, pore size, and permeability, and the grain parameters are density and bulk modulus. The frame parameters are the bulk and shear moduli (real and imaginary parts). Obtaining practical values for the several poroelastic parameters is an unsolved problem that is approached with a combination of empiricism and theoretical approximation.

The acoustic theories described above assume marine sediment is a single-phase (fluid or solid) or two-phase (fluid-water) medium. In fact, sediments are quite complex assemblages of a variety of particle types, pore fluid, organic matter, and, sometimes, free gas. Even a small amount of free gas can greatly alter sediment compressional wave speed (compressibility), compressional wave attenuation, and acoustic scattering. However, sediment density and shear wave speed and attenuation are not affected as much by small amounts of free gas.

\section{Sea bottom modeling using fluid theory}

The term "fluid theory" refers to system of equations governing wave motion and boundary conditions for sound pressure fields' (and related fields) in sediments. Basing on the theory is elaborated the model which is application of theory with simplifying approximations [5].

The acoustic modeler will usually choose the simplest theory that can account for the phenomena evident in the data at band. The parameters of interest when applying the fluid theory are the sound speed, attenuation, and bulk density of sediment and seawater. Methods to measure sediment bulk density in the laboratory and in situ as well as sound speed and attenuation measurement techniques are at present generally used in the sea investigations. Empirical regressions between these parameters and commonly measured sediment physical properties (such as mean grain size and porosity) are also given [3]. Empirical relations to predict the relevant parameters of the water column in terms of temperature, pressure, and salinity are given in [6].

Although sediments are composed of discrete particles, the theory treats sediments as continuous media. This approximation is reasonable provided the wavelength is much larger than the size of the sediment grains [4]. The theory of interest can be treated as effective medium theory in which physical properties such as density and elastic moduli are spatial averages of same sort. The term "bulk density" is a reminder of this averaging and serves to distinguish sediment density from the constituent grain and water densities.

Fluids and solids are treated as continuous media even though they are composed of discrete atoms, molecules, and/or crystal grains. For example, a quartz crystal is often described in terms of elastic moduli, optical refractive index, etc., without concern for its underlying atomic structure.

The discrete structure of sediments will become apparent acoustically when the acoustic wavelength becomes comparable to sediment particle size, particle separation, or pore size. For the acoustic frequencies of interest, wavelengths (in water) range from about $15 \mathrm{~cm}$ to $1.5 \mathrm{~mm}$. As the coarser sand sediments have grain sizes of order $1 \mathrm{~mm}$, the continuum treatment should be adequate except at the highest frequencies for the coarser sediments.

This picture implies averaging over a volume containing many sediment particles, but doesn't clearly specify the size of this averaging volume. If the averaging volume is too small, containing only a few particles, the resulting average density and modulus may have an extreme variation with position and may not be physically meaningful. On the other hand, if the averaging volume is chosen to be several acoustic wavelengths in size, the heterogeneity responsible for acoustic volume scattering will be smoothed out and lost. Thus, the idea of an effective medium is not as clear as it should be, and the success of this approach is conditional (as previously noted) on the acoustic wavelength. It will be assumed that geoacoustic measurements provide averages over suitable volumes, neither too small nor too large for the acoustic problem at hand. However such optimistic view is not always justified, that is, the averaging volume in geoacoustic measurements may be too large for the acoustic application. One can believe, however, that as long as the acoustic wavelength is much larger than the sediment particle size, suitable averaging should be possible in principle, if not always in practice.

\section{Results of modeling}

The parameters used in modeling the phenomena of transmission and reflection of sound wave into and from marine sediments were assumed to be comparable to those used in the measurements. The frequency of a 
plane wave incident perpendicularly towards bottom was from range $4-15 \mathrm{kHz}$. The shape of sampling pulse was identical to pulse transmitted by the echosounder.

The bottom was assumed to be composed of few layers characterized by geophysical properties which are typical for real sediments (Fig. 2). Accordingly to the conditions of experiment, it was assumed that the wave incidents perpendicularly at a water — sediment border, while a part of acoustic energy reflects from the border, and the other part transmits into the upper layer of the bottom sediments appropriately to the acoustic impedances quotient of both media. The sound penetrating into the sea bottom encounters successive layers of sediments and each border between them is a place of reflection and transmission of the acoustic energy.

The main parameters characterizing the sound propagation in sediments are given in the dimensionless form compared to adequate parameters of sea water and are as follows:

— dimensionless sound speed:

$$
a_{p}=\frac{c_{p}}{c_{w}}
$$

— dimensionless density:

$$
a_{p}=\frac{\rho_{p}}{\rho_{w}}
$$

where $c$ is compressional wave phase speed, $\rho$ is density, index $p$ denotes sediment, and index $w$ denotes water,

- and attenuation coefficient:

$$
a_{b}=k_{p} f_{a}^{m}
$$

where $k_{p}$ is an attenuation constant expressed in $\mathrm{dB} \mathrm{m}^{-1} \mathrm{kHz}^{-1}$ and $f_{a}$ is the acoustic frequency in $\mathrm{kHz}$. According to Hamilton's observation for unconsolidated marine sediments $m=1$, and coefficient $k_{p}$ is relating to the mean grain size [4]. The values of those parameters for chosen type of sea bottom sediments are given in Table.

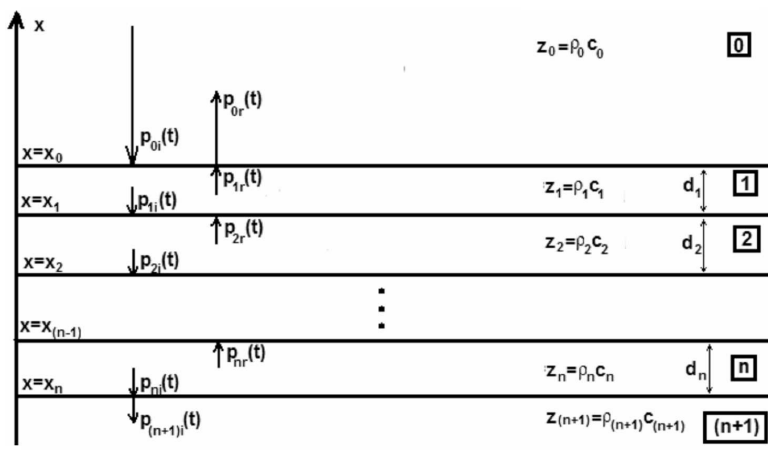

Fig. 2. Sound beam penetrating into layered sea bottom.

In the model investigation it was calculated the shape of echo obtained for different type of bottom structure. The geoacoustic parameters, as well as the thickness of particular layers was changed. Besides, the infuence of duration and frequency of penetrating pulse on image of sediments was examined. In the following two figures are presented the results obtained for bottom structure that could appear in the Baltic Sea. The first structure is composed of three layers, each of 1 meter thikness: medium silt, coarse silt and find sand based on the bottom from medium sand. The second structure is composed of four layers: clay, fine silt, fine sand and coarse sand based on the bottom from fine gravel.

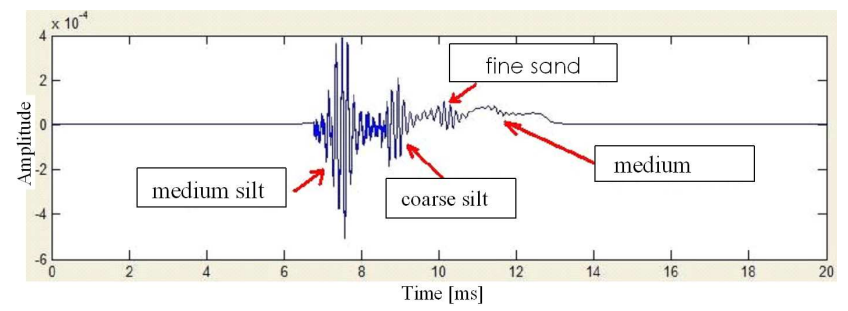

Fig. 3. The pulse reflected from a marine bottom composed of four layers. Top layer - one meter thick medium silt, second layer - coarse silt, third layer fine sand and the bottom layer is medium sand.

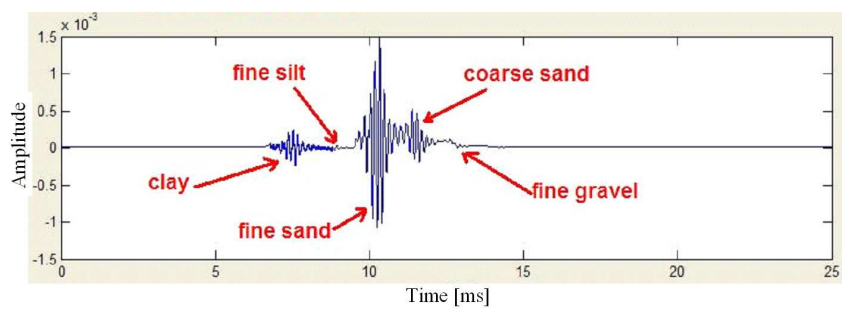

Fig. 4. The pulse reflected from a marine bottom composed of following layers: clay, fine silt, fine sand, coarse sand and the bottom layer is medium sand.

Given examples illustrate the problem that appears in the interpretation of the results obtained during in situ measurements. Namely, the amplitude of the echo from the first border (water - upper layer of bottom sediments) is not the largest one. The amplitude of the echoes from successive layers depends on their geoacoustic parameters. In the natural sea conditions different composition of sea bed sediments could be observed. The first case (Fig. 3) illustrates situation when the upper layer is of rather great acoustic impedance and as a consequence, the echo from the border allows to determine precisely and unequivocally the depth of the sea and the consecutive layers. The second example (Fig. 4) shows the bottom structure more complicated for interpretation. The layers of sediments with geoacoustic parameters more similar to those of sea water laying on the layer of higher acoustic impedance can cause the image when the echo from the third layer is greater than the previous ones. However, such images are observed in natural conditions. 


\section{Conclusions}

The paper presents results of modeling marine bottom structure basing on the fluid theory. The model which is application of theory with simplifying approximations has been elaborated and examined. The main goal of the investigation is to obtain the tool that can be used for interpretation data collected by parametric echosounder. Nevertheless the model has a lot of simplifications, the preliminary results seem to be promising.

\section{Acknowledgments}

The investigation was supported by the Ministry of Science and Higher Education (Grant No R00 012 01).

This paper is mainly based on the paper: "Modeling of Sounding Layered Marine Bottom" presented during 16 ICSV2009 in Cracow.

\section{References}

[1] G. Grelowska, E. Kozaczka, Hydroacoustics 11, 105 (2008).

[2] E.L. Hamilton, Geophysics 37, 620 (1972).

[3] Ł. Nowak, W. Szymczak, Hydroacoustics 12, 189 (2009).

[4] G. Grelowska, E. Kozaczka, Modeling of sounding layered marine bottom, Proceedings of the XVI Int. Congress on Sound and Vibration, Kraków, 5-9 July 2009, 6 pp. CD.

[5] D.R. Jackson, M.D. Richardson, High-Frequency Seafloor Acoustics, Springer, 2007.

[6] N.P. Fofonoff, R.C. Millard Jr., Algorithms for computation of fundamental properties of seawater, Unesco technical papers in marine science, No 44, UNESCO, 1983.

[7] D.D. Sternlicht, Ch.P. Moustier, J. Acoust. Soc. Amer. 114, 2709 (2003). 